\title{
Route Choice in Subway Station during Morning Peak Hours: A Case of Guangzhou Subway
}

\author{
Jie Xu, ${ }^{1}$ Yao Ning, ${ }^{2}$ Heng Wei, ${ }^{3}$ Wei Xie, ${ }^{2}$ Jianyuan Guo, ${ }^{2}$ Limin Jia, ${ }^{1}$ and Yong Qin ${ }^{1}$ \\ ${ }^{1}$ State Key Laboratory of Rail Traffic Control and Safety, Beijing Jiaotong University, Beijing 100044, China \\ ${ }^{2}$ School of Traffic and Transportation, Beijing Jiaotong University, Beijing 100044, China \\ ${ }^{3}$ Department of Civil \& Environmental Engineering, University of Cincinnati, Cincinnati, OH 45221, USA
}

Correspondence should be addressed to Jie Xu; xujie@m.bjtu.edu.cn

Received 18 July 2014; Accepted 1 September 2014

Academic Editor: Yongjun Shen

Copyright (C) 2015 Jie Xu et al. This is an open access article distributed under the Creative Commons Attribution License, which permits unrestricted use, distribution, and reproduction in any medium, provided the original work is properly cited.

\begin{abstract}
This paper is aimed at crowding phenomenon in the subway. As passengers are inclined to choose the route with minimum disutility, we put forward a route choice model which is constructed to achieve minimum objective function of feasibility for the optimal solution. Meanwhile we set passenger volume threshold values according to capacity of facilities. In the case of actual capacity exceeding the threshold, the decision node of constrained route will be selected; computing procedure about searching decision points will be presented. Then we should set rational restrictions at the decision node of the minimum utility function route to prevent too many passengers' access to platform. Through certification, this series of methods can effectively ensure the safety of the station efficient operation.
\end{abstract}

\section{Introduction}

Due to heavily overcrowded population and situations of underground space, a lot of potential risks exist during operation of subway system in most of metropolitans of China. More and more incidents (even accidents) occur in subway systems worldwide, endangering the safety and security of subway's operation. To prevent overcrowding of trains and platforms at peak hours, queuing palisades are installed out of congested stations. Thus a certain proportion station gates are closed and commuters have to detour the palisade to enter station at peak hours. It costs much more time to access station, and too many passengers stagger and detour alone the palisade to enter stations during peak hours. And it has been a routine measurement of subway station operation in Beijing, Shanghai, and Guangzhou (Figure 1). There are big concerns on such measures due to safety reasons and complaints of the commuters. As of May 12, 2014, twenty-five stations, mainly on Line 1, Line 3, Line 4, Line 5, and Line 8 in Beijing, have taken such measures. Some of these stations have built queuing obstacles outside stations to keep passengers from entering stations. The restrictive measurements are put in use during 7:45-9:30 AM peak hours. Moreover, the schemes for limiting passenger crowding had been issued by Shanghai Subway Co., and passenger flow limiting measures are in action at more than thirty stations. The passenger limiting schemes mentioned above are designed by staffs of station according to their experience. However, how do we make an appropriate scheme for a given station according to passenger behaviors? And how much capacity of specified facilities should be constrained to avoid leading to stampede because of overcrowded passenger? In order to deal with the issues above, this paper takes into account both transfer station layout and passenger movement behavior at peak hours. The following problems are discussed: (1) network building for station layout; (2) determination of route choice in subway station when facility capacity is insufficient. This preliminary work paves the way for the development of a methodology, which can be used for designing schemes of passenger flow limiting for subway managers and staffs of transfer station at rush hours. 


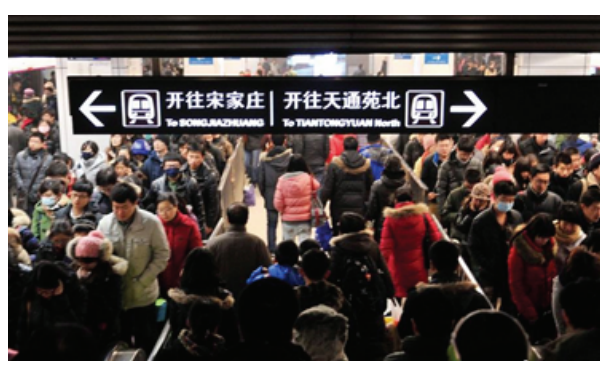

(a) Transfer passengers increase significantly along with new line being put into use

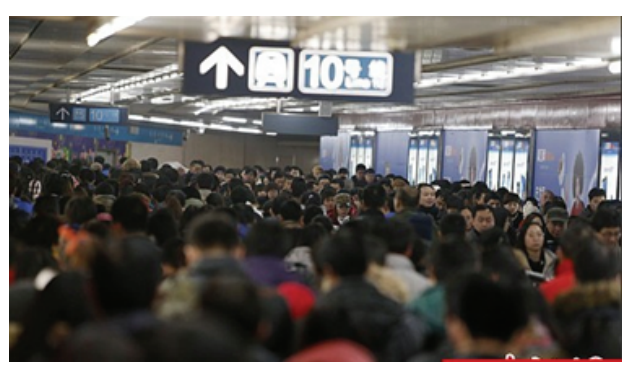

(b) Passage transfer capacity of station do not meet the demand

FIgURe 1: Overcrowding in subway transfer station.

\section{Related Works}

Since April 2011, seven stations have imposed such restrictions in Beijing Subway Co. Some of these stations have built up queuing palisades outside to prolong passengers' entering time and reduce passenger volume during morning peak hours. Similarly, the schemes of preventing passenger overcrowding had been issued by Shanghai Subway Co., and the measures have been in action at more than thirty stations since 2010. Moreover, the actions have been implemented in Guangzhou Metro Co. since November 2011. In the following sections an overview is given of the main literatures with respect to influencing factors on route choice and pedestrian behaviors in railway station. Transfer passengers are evenly distributed over the different routes in the station, which does not only consider walking time (including delays in front of and on escalators and stairs) and walking distance but also considers the effort involved in climbing a grade [1]. And bridging level variation (ramps, stairs, and escalator) has a significant and different impact on the attractiveness of a route for a traveler [2]. Daamen [3] focused on empirical data and modelling of pedestrian route choice and investigated influencing factors of route choice in railway stations, which may be assembled network characteristic, route characteristics, and pedestrian characteristics.

Models for pedestrian behavior stimulation have been developed to evaluate aggregation of pedestrian flows at various facilities, such as railway and subway stations. Generally speaking, the approaches of modelling pedestrian's movement are divided into two main groups: microscopic and macroscopic models [4-9]. The studies from the perspective of microscopic mainly focus on the behaviors of individuals and consider their interference between pedestrians during walking $[10,11]$. Relationships between single direction pedestrian speed and density were studied based on regression analysis. The main models include cellular automata mode, lattice gas model [6, 12-14], and social force model $[7,15,16]$. The crowd was regarded as a self-organizing system with cellular automata, which was composed of many individuals moving in station facility network according to certain rules, movements, and decisions under normal and emergency condition. The station reliability and design effect of facilities were evaluated [17]. Hoogendoom and Daamen [18] proposed a model of station space and passengers walking and adopted microscopic simulation tools to simulate the distribution of passengers on platform. Daamen [3] built three hierarchies of pedestrian dynamics model and crowds modeling system to evaluate design of passage, walkway, stairs, and so on. Then, mass movement and distribution in complicated space were stimulated. The behaviors of passengers in station during peak period were discussed. Analytic function about passengers' walking time on nine kinds of pedestrian facilities was achieved. Pedestrian walking model was established based on the walking time [19].

Several studies have analyzed pedestrian route choice in outdoors and closed space, as railway and subway stations. Lee et al. [19] investigated behaviors of pedestrians in Hong Kong Mass Transit Railway (MTR) stations during peak hour periods. As the pedestrian walking behavior would be influenced by the physical properties of the walking facilities, nine pedestrian facilities are classified for analysis. Surveys were conducted in the Hong Kong Causeway Bay MTR Station. The data collected were used to estimate passengers Origin-Destination (O-D) flow matrix within the station. Cheung and Lam [20] used binomial logit model to simulate Hong Kong subway passengers' behaviors. Their choices between adjacent escalators and stairs based on the difference of walking time were discussed. Lam and Cheung [21] focused on findings of pedestrian flow characteristics for different types of walking facilities in Hong Kong. Hoogendoorn and Bovy [22] put forward an approach for useroptimal dynamic assignment in continuous time and space. The approach consists of three interrelated steps, that is, determining continuous paths using a path choice model, assigning origin, destination flows, and calculating resulting of traffic conditions. Hoffman et al. [23] forecasted probability of all alternative path choices from origin to destination and the process was divided into multiple stages. [24] established passenger delay model of rail station stairs and escalators on queuing theory and the index formula of passenger delays. And the relationship between pedestrian flow and facilities was obtained, travel time function based on different pedestrian facilities was calibrated, and route choice behaviors of passengers in station were discussed [25].

To avoid overcrowding at platform during peak hours, constrained routes will be designed by adjusting the capacity of facilities (i.e., passages, stairs, entrance, and so on) to 


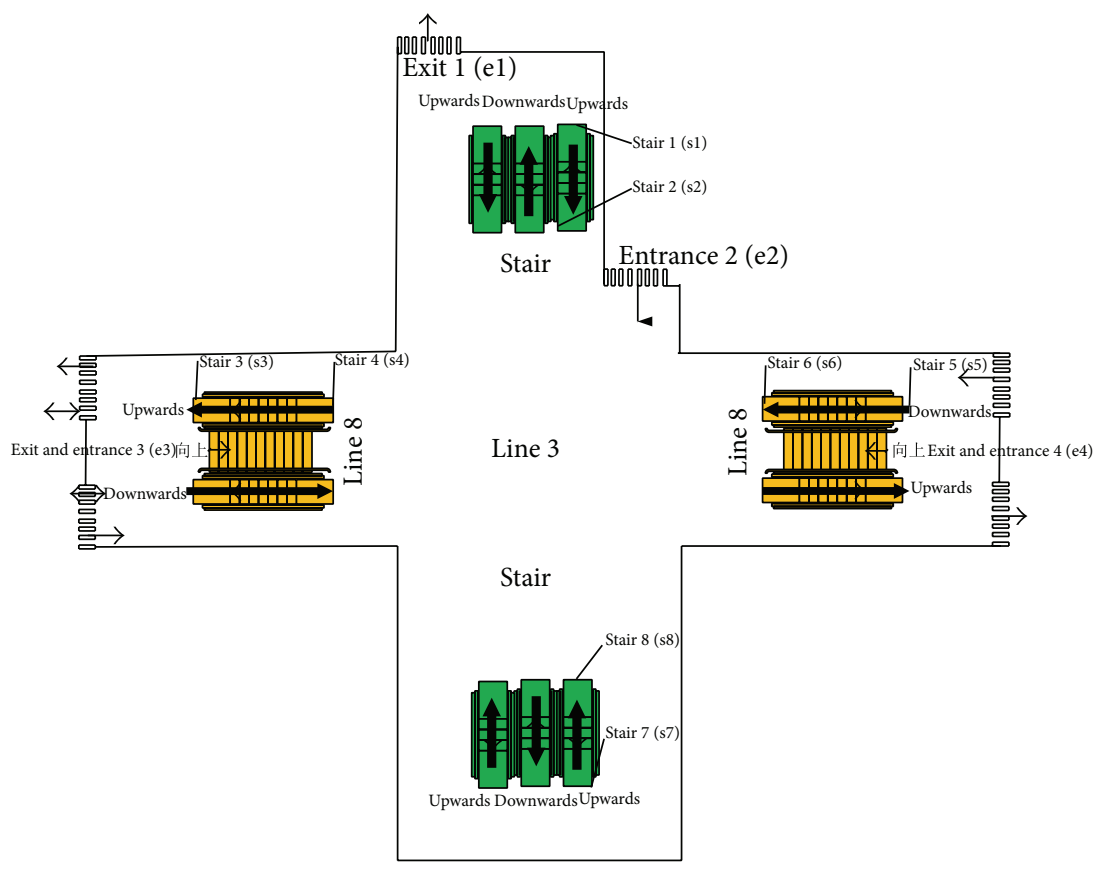

FIGURE 2: Layout of first floor of Kecun station.

prolong walking time of transfer passengers. So that entering and transfer passengers will be kept outside, concourse, transfer passages and other transfer facilities longer. However, how do we design specified routes to avoid passenger overcrowding at stations bottleneck? A route choice model will be constructed to illustrate the question. As a typical transfer station of two lines, the methods and measurements in Kecun station of Guangzhou Subway will be presented.

\section{Dimension of Station}

Kecun station is a transfer station and has three transfer level structures, locating at junction of Line 3 and Line 8 in the Guangzhou subway network. Similar structure exists at several stations in Beijing The layout of Kecun station is shown in Figure 2. A route consists of a series of facilities, each of which indicates exact spatial path that pedestrians follow within a specific area (i.e., exit, passage, platform, hall, stair, escalator, and entrance), as Figure 3 shows. Routes for passengers consist of such series of subsequent facilities (entrances $\rightarrow$ gates of Automatic Fare Collection (AFC) $\rightarrow$ concourse passages $\rightarrow$ stairs or escalates $\rightarrow$ passages $\rightarrow$ platform $\rightarrow$ shielded gates). Transfer passengers go from the shielded gates of alighting platform and then go through transfer facilities (passages and stairs or escalates) to shielded gates of boarding platform of another line. Escalator $(1,2)$ and escalator $(7,8)$ are vertical facilities connecting the first station level concourse and platform of Line 3 , which are colored by dark green. Stairs $(3,4)$, escalator $(3,4)$, escalator $(5,6)$, and stairs $(5,6)$ connect the concourse, and the platform of Line 8 is marked with yellow color. The light green stairs are transfer stairs of Line 3 to connect transfer passages and platform, and purple ones are also transfer stairs, which is to link transfer passages and platform of Line 8 .

For simplicity, station structure is assumed as network $G=(N, E)$. Because it is easy to adjust pedestrian flow capacity at joints of facilities, the joints are considered as nodes of network $n \in N$, and exits, shielded gates, and entrances (entrances and exits, exits) are defined as the source node $s \in N$ and the sink node $t \in N$, respectively. Edge ( $\operatorname{arc})$ is an arc between two nodes. Each edge $e \in E$ has an associated capacity $u_{e}$ and a walking time of pedestrians (or length of facilities) through the facilities $t_{e} \geq 0$. The walking time on overlap zone of various facilities, such as entrances (exits and entrances) and gates of Automatic Fare Collection (AFC) are ignored because of their minute sizes. The walking network of station is depicted as Figure 4. As usual, pedestrians arrive at the platform along the color lines because of their minimum utility function.

\section{Model}

4.1. Route Choice. The selected cost of facility is represented as utility when passengers traverse the facility. The utility function can be estimated by walking and waiting time on different types of infrastructure and the overlap factors. Because route and path capacities are constrained by size joint of adjacent facilities causing crowdedness easily, the joints, such as gate of AFC and passage (concourse), stairs (escalator), and concourse (passage), are regarded as decision points. And for the points, width of the joint will be adjusted to decrease capacity, and fewer passengers will entry the facility to reduce its crowdedness. The route choice is considered as dynamic procedure, and several concepts related to the approach are involved [26]. Firstly, these concepts are defined as follows. 


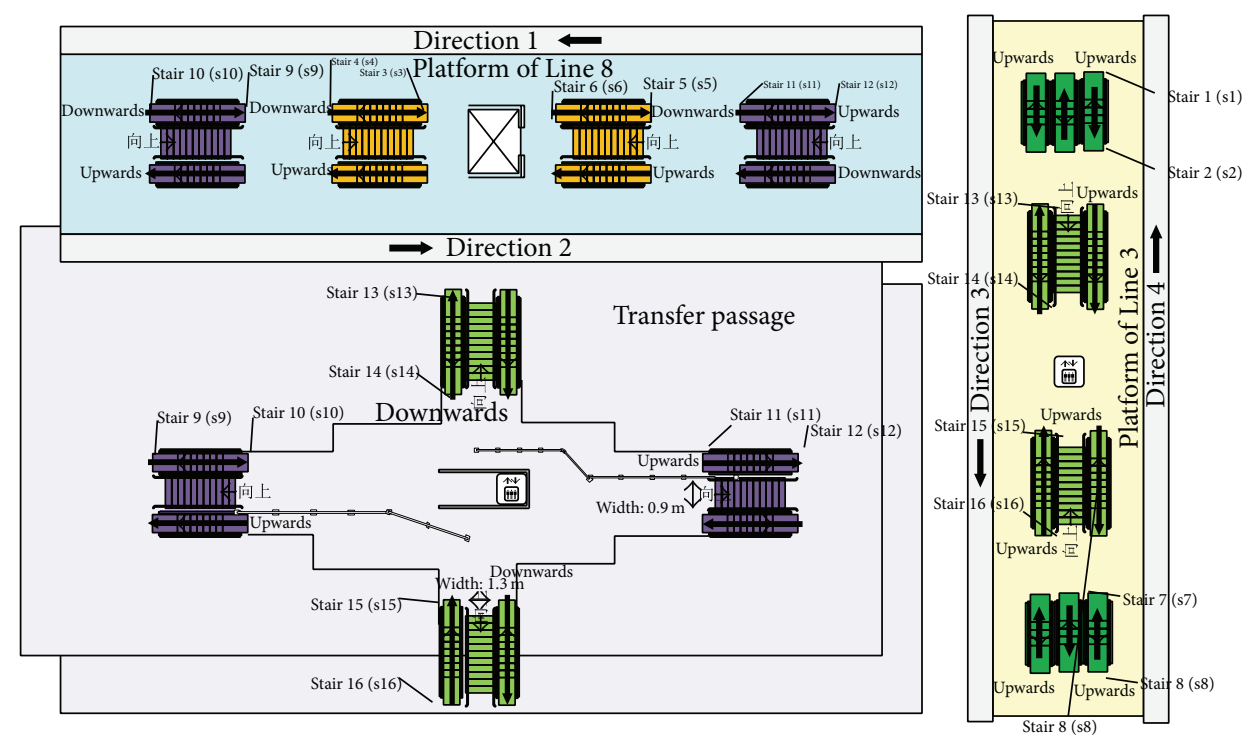

Figure 3: Transfer structure network of Kecun station.

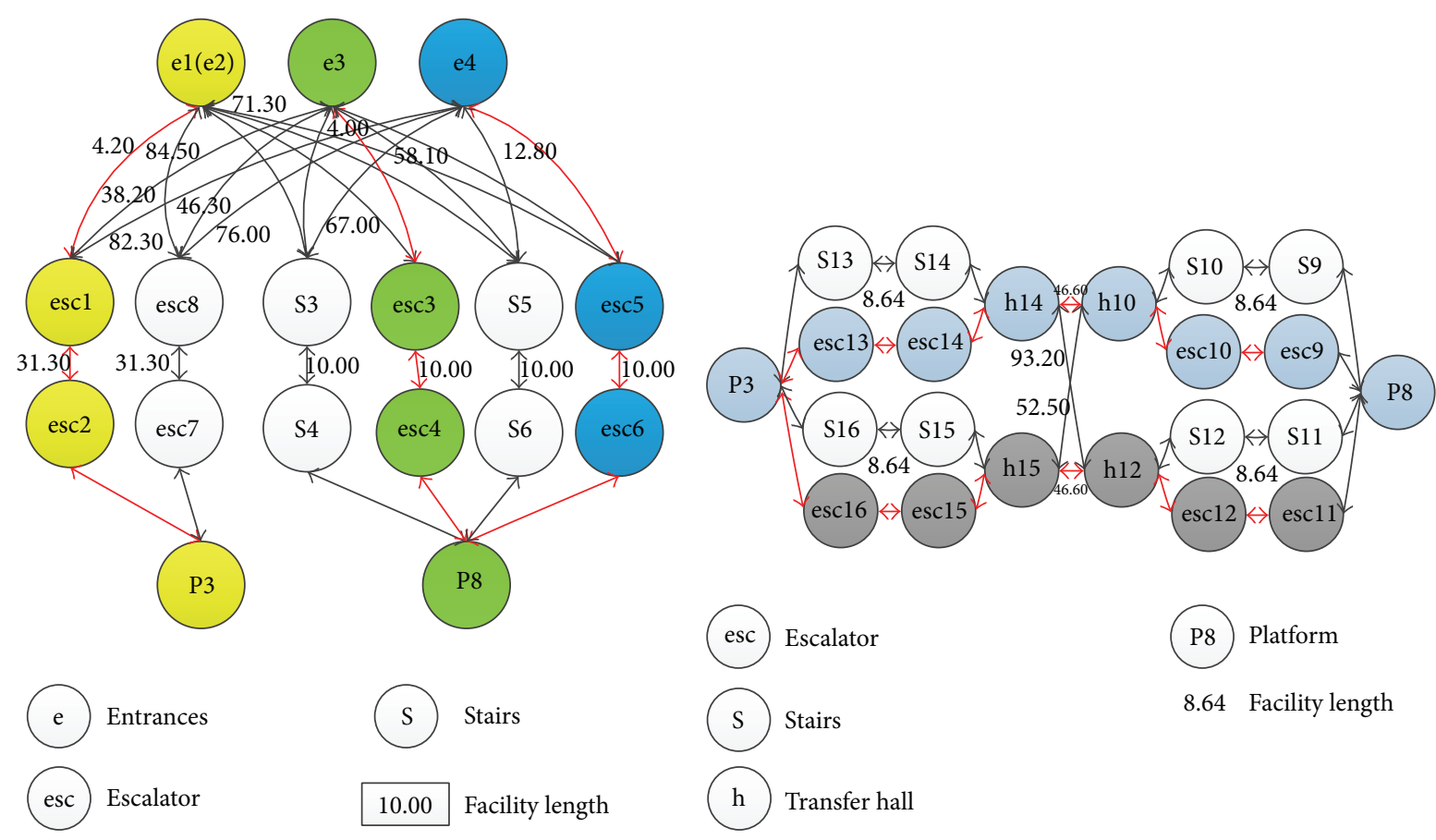

(a) Passenger inbound walking network

(b) Passenger transfer walking network

FIGURE 4: Walking network structure of Kecun station.

Assume that $S_{k}=\left\{N_{k}, E_{k}\right\}$ is optional set of facilities in the station at $k$ th route-choice stage, $N_{k} \subset N$ is alternative node of next stage, and $E_{k} \subset E$ is alternative edge of next stage.

Definition 1. A utility function of selecting node $A_{k}\left(i, j, t_{j}\right)$ is utility function of selecting node $j$ at node $i$, and $t_{j}$ is passenger waiting time at joints in front of node $j$ because of crowding. It can be assumed as

$$
A_{k}=\mu \sum D_{i, t_{j}}
$$

in which $D_{i, t_{j}}$ is the time cost when node $j$ is chosen at node $i, \mu=$ coefficient for calibration.

However, opposite direction passengers might cause facility capacity to be reduced, and bidirectional facilities have impact on trajectory selection. Thus, the transfer passages and stairs are considered as conflict nodes in the paper. 
TABLE 1: Travel time functions of pedestrian facilities.

\begin{tabular}{lccccr}
\hline \multirow{2}{*}{ Pedestrian facility } & \multirow{2}{*}{ Number of samples } & \multicolumn{2}{c}{ Parameters } & Facility capacity (peds/m/min) & $\begin{array}{l}\text { Walking speed at } \\
\text { capacity (m/min) }\end{array}$ \\
\hline Escalator (ascending) & 548 & 0.2502 & 1.1732 & 120 & 54.01 \\
Stairway (ascending) & 698 & 1.1405 & 2.9158 & 70 & 51.36 \\
Stairway (descending) & 662 & 14.24 & 1.742 & 80 & 58.28 \\
Passageway & 718 & 26.5277 & 0.5985 & 92 & 0.8057 \\
\hline
\end{tabular}

Definition 2. A conflict point $c_{p}$ is a bidirectional facility in the subway station, because pedestrian would be interfered with each other while walking on the facility at peak hours. There are many conflict nodes on the hall, concourse, and platform of transfer stations. That is to say, if passage or stairs are bidirectional, conflict point is 1 ; otherwise, it is 0 .

Definition 3. A control coefficient is a function to be evaluated if passenger volume accumulates to maximum facility capacity. When the passenger density at the facility surpasses the capacity threshold, measurements should be taken to decrease passengers' entry rate. Therefore, passenger volume control coefficient on the trajectory $j$ is defined as follows:

$$
C_{j}= \begin{cases}0, & \rho_{j} \leq \rho_{c} \\ 1, & \rho_{j} \leq \rho_{c}\end{cases}
$$

Obviously, if $C_{j}=1$ the utility function above will be increased.

Walking time at walking facility of station has relationship with passenger density; that is to say, crowdedness is often indicated as being important in pedestrian route choice; path with low pedestrian density tends to be chosen. The equation adopted for estimating the travel time is based on the wellknown Bureau of Public Roads (BPR) function [21]. The BPR travel time function by pedestrian facility is given as follows:

$$
t(V)=t_{0}+B \times\left(\frac{V}{C}\right)^{n}
$$

where $C$ is capacity of pedestrian facility (peds $/ \mathrm{m} / \mathrm{min}$, peds/esc/min, or peds $\left./ \mathrm{m}^{2}\right) ; V$ is pedestrian flow or density (peds $/ \mathrm{m} / \mathrm{min}$, peds/esc/ min, or peds $\left./ \mathrm{m}^{2}\right) ; t(V)$ is travel time (s) at flow $V$, and $t_{0}$ is free-flow travel time (s); $B$ and $n$ are parameters to be estimated. Through field investigation, the travel times and the corresponding flows were obtained. And the results for different facilities were converted to speed/flow relationships which were used to calibrate the travel time function for each pedestrian facility. Six Beijing subway stations were chosen for calibrating the parameters $B$ and $n$. The observed capacities for the various types of pedestrian facilities were obtained and tabulated in Table 1 in workdays of two months.

Definition 4. A walking utility function of selecting facility $W_{k}\left(n_{i}, n_{j}, e_{k_{m}}\right)$ is utility function of selecting facility $e_{k_{m}}$ from node $i$ to $j$. In subway stations, route length or walking time plays a role in the route choice process. Therefore, (3) is used to depict walking utility function. During peak hours, utility value of walking function is dynamic variables and is determined by facility capacity, passenger density, speed, and conflict point. So, the walking utility function of facility is depicted as follows:

$$
W_{k}\left(n_{i}, n_{j}, e_{k_{m}}\right)=\alpha \cdot t_{k}\left(n_{i}, n_{j}, e_{k_{m}}\right)+\theta c_{p}+\delta C_{e_{k_{m}}} .
$$

Refernce [26] estimated $\theta=10, \delta=1000$. Therefore,

$$
W_{k}\left(n_{i}, n_{j}, e_{k_{m}}\right)=\alpha \cdot t_{k}\left(n_{i}, n_{j}, e_{k_{m}}\right)+10 c p_{k_{m}}+1000 C_{e_{k_{m}}} .
$$

Based on collected data of Dutch train stations and a multinomial path-size logit model, a utility function has been put forward, in which not only walking time and a route overlap factor are taken into account but also the influences of level changes in routes are included. So the utility function to be estimated consists of walking times on different types of infrastructure and the overlap factor [2]:

$$
\begin{aligned}
U_{r}= & -0.130 T_{r}^{l}-0.242 T_{r}^{\mathrm{st}}-0.167 T_{r}^{\mathrm{esc}} \\
& -0.1787 T_{r}^{\mathrm{ramp}}+3.181 P S_{r},
\end{aligned}
$$

in which $U_{r}$ is utility of route $r, T_{r}^{l}$ is walking time on level elements of the infrastructure that are part of route $r, T_{r}^{\mathrm{st}}$ is walking time on stairs in route $r, T_{r}^{\mathrm{esc}}$ is walking time on escalators in route $r, T_{r}^{\text {ramp }}$ is walking time on ramps in route $r$, and $P S_{r}=$ path size of route $r$.

And utility function of route $R_{k}$ is formulated as follows:

$$
U_{R_{k}}\left(O, D, R_{k}\right)=\sum_{i=1}^{N_{k}} A_{k}(i, j)+\sum_{e_{k_{m}}=1}^{E_{k}} W_{k}\left(n_{i}, n_{j}, e_{k_{m}}\right),
$$

in which $O=1, D=N_{k}$. Therefore,

$$
\begin{aligned}
U_{R_{k}}\left(O, D, R_{k}\right)=\sum_{i=1}^{N_{k}} A_{k}(i, j) & \\
-\sum_{k_{m} \in E}( & 0.130 T_{k_{m}}^{l}+0.242 T_{k_{m}}^{\mathrm{st}}+0.167 T_{k_{m}}^{\mathrm{esc}} \\
& +0.1787 T_{k_{m}}^{\mathrm{ramp}}+3.181 P S_{k_{m}} \\
& \left.-10 c p_{k_{m}}-1000 C_{k_{m}}\right) .
\end{aligned}
$$


TABLE 2: Average inbound passenger flow density at facility during peak hours.

\begin{tabular}{lcccccccc}
\hline & $7: 15-7: 30$ & $7: 30-7: 45$ & $7: 45-8: 00$ & $8: 00-8: 15$ & $8: 15-8: 30$ & $8: 30-8: 45$ & $8: 45-9: 00$ & $9: 00-9: 15$ \\
\hline Line 3 & 0.64 & 0.93 & 1.48 & 1.62 & 1.51 & 1.43 & 1.29 & 0.97 \\
Line 8 & 0.819 & 1.168 & 1.82 & 2.37 & 2.47 & 2.60 & 2.30 & 1.61 \\
\hline
\end{tabular}

Then, because overcrowdedness of station platform might lead to passenger stampede or fall into the rail leading to being wounded or fatal, extending walking time in the station through facility capacity is restricted to decrease pedestrian density on the platform. Therefore, the route with maximum path-size will be chosen for subway stuffs to decrease passenger flow arriving at platform during unit period at rush hours. The restricted major problem is formulated as follows:

$$
Z=\operatorname{minimise} \sum_{k=1}^{n} U_{R_{k}}\left(O, D, R_{k}\right) .
$$

That is to say, when passenger flow is too crowded, the routes with minimum utility function have to be closed or reduced capacity to increase utility or waiting time of the routes and decrease passenger density at the platform. Therefore, the restricted route has to be chosen according to utility function, and then decision points, which are specified facilities and regulated capacity, will be determined.

\section{Methodology of Determination of Decision Points}

Firstly, some conceptions are defined as follows:

sep: set of sequence facilities in which passenger volume is prone to amount the capacity threshold;

ses: set of routes which consist of elements of sep;

seq: set of decision nodes.

There are several passenger flow lines in transfer station, such as passenger input line, output line, and transfer line. Since passenger flow in the station varies, the decision points of route mentioned above should be changed. That is to say, alternative routes are determined based on given passenger volume of all kinds of passengers (such as inbound passengers, outbound passengers, and transfer passengers). Then, passengers are assigned to the initial restricted route. The passenger volumes in various facilities are reviewed again, if they exceed the capacity threshold of the facilities, respectively, the utility of decision points have to be enhanced or increased according to passenger volume. The detailed steps of determining decision nodes are as follows.

Step 1. Calculate every facility designed capacity and building feasible route set bounding of the platform. Search the facility with minimum capacity and establish the set of sep.

Step 2. Determine the trajectory sequence of ses based on set of sep.
Step 3. Check the trajectory sequence of ses; the route with minimum utility function will be chosen to alternative route. If corresponding nodes can be set as decision node in the sep, go to Step 5; otherwise, go to Step 4.

Step 4. Search set of route in the ses and identify if node of prior stage can be set as decision node; go to Step 5; otherwise, repeat Step 4.

Step 5. If there is outbound route between accessed nodes, the decision nodes on the outbound route are removed. If one decision node is the one on inbound route and another is on transfer route, the passenger volume restricted measures will be put in effect on the inbound route. If both decision nodes are on transfer route, utilities of nodes $U_{R_{k}}\left(O, D, R_{k}\right)$ are calculated and compared. The higher one is selected as decision node to be set as sep.

Therefore, the decision points are set prior to the facility with minimum capacity of minimum utility function route; passenger flow will spend more time on entering station. This will ensure that passenger volume would not surpass platform capacity at rush hours, if the capacity of decision points is adjusted properly.

\section{Example}

6.1. Data. Layout of Kecun station and facilities dimension are shown in Figure 3. There are 4 routes and 2 routes from the entrance to the platform of Line 3 and Line 8 , respectively. The facilities parameters are shown in Table 2; the effective area of platform of Line 3 and Line 8 is $568.8 \mathrm{~m}^{2}$ and $635.6 \mathrm{~m}^{2}$, respectively. The train's rated passenger capacity is 1860 person/train, and service frequency is 3 minutes during peak hours.

Inbound passenger flow can be achieved from AFC every 15 minutes. Therefore, passenger flow density at entrance will be computed and tabulated in Table 2. The passenger flow speed and travel time will be calculated by (3).

According to study of $\mathrm{Bi}$ and Jiang [27], the critical density threshold of platform is $0.5 \mathrm{~m}^{2} /$ peds, platform of Line 8 is overcrowded during peak hours. The inbound passenger flow constrained measurements have to be put in use.

Because escalator, staircase, and platform are bottleneck of station $[21,28]$, passengers on the facility are more crowded than those on passages. Passengers have to come to halt because it is too crowded during rush hours. The waiting time of various time intervals during peak hours is shown in Table 4.

In Table 3, esc ${ }_{1}, \operatorname{esc}_{8}(D)$ means the waiting time at nodes $\operatorname{esc}_{1}$ and esc $_{8}$ (beginning of escalator of Line 3 ) and going 
TABLE 3: Passenger volumes at the platforms.

\begin{tabular}{lcccccccc}
\hline Facility & Effective area $\left(\mathrm{m}^{2}\right)$ & $7: 30-7: 45$ & $7: 45-8: 00$ & $8: 00-8: 15$ & $8: 15-8: 30$ & $8: 30-8: 45$ & $8: 45-9: 00$ & $9: 00-9: 15$ \\
\hline P3 & 535.6 & 468 & 566 & 588 & 518 & 425 & 361 \\
P8 & 468.8 & 417 & 773 & 1148 & 1007 & 907 & 756 & 706 \\
\hline
\end{tabular}

TABLE 4: Waiting time in different time interval during peak hours.

\begin{tabular}{lccccccc}
\hline Point & $7: 30-7: 45$ & $7: 45-8: 00$ & $8: 00-8: 15$ & $8: 15-8: 30$ & $8: 30-8: 45$ & $8: 45-9: 00$ & $9: 00-9: 15$ \\
\hline $\operatorname{esc}_{1}$, esc $_{8}$ (D) & 20 & 22 & 22 & 22 & 22 & 22 & 14 \\
$\operatorname{esc}_{3}, \operatorname{esc}_{5}$ (D) & 19 & 22 & 23 & 23 & 23 & 22 & 15 \\
\hline
\end{tabular}

TABLE 5: Utility functions of routes.

\begin{tabular}{lccc}
\hline Route & Waiting time $(\mathrm{s})$ & Walking time $(\mathrm{s})$ & Utility function \\
\hline$e_{3} \rightarrow \mathrm{esc}_{3} \rightarrow \mathrm{esc}_{4} \rightarrow P_{8}$ & 23 & 113.97 & 135.97 \\
$e_{3} \rightarrow \mathrm{esc}_{5} \rightarrow \mathrm{esc}_{6} \rightarrow P_{8}$ & 23 & 104.69 & 127.69 \\
$e_{4} \rightarrow \mathrm{esc}_{3} \rightarrow \mathrm{esc}_{4} \rightarrow P_{8}$ & 23 & 116.08 & 138.08 \\
$e_{4} \rightarrow \mathrm{esc}_{5} \rightarrow \mathrm{esc}_{6} \rightarrow P_{8}$ & 23 & 65.26 & 88.26 \\
$e_{3} \rightarrow s_{3} \rightarrow s_{4} \rightarrow P_{8}$ & 23 & 110.45 & 145.23 \\
$e_{3} \rightarrow s_{5} \rightarrow s_{6} \rightarrow P_{8}$ & 23 & 112.34 & 148.28 \\
$e_{4} \rightarrow s_{3} \rightarrow s_{4} \rightarrow P_{8}$ & 23 & 109.36 & 140.90 \\
$e_{4} \rightarrow s_{5} \rightarrow s_{6} \rightarrow P_{8}$ & 23 & 110.23 & 142.59 \\
\hline
\end{tabular}

downward from node esc 1 to node $\mathrm{esc}_{2}$. And esc $\mathrm{esc}_{3}(D)$ means the waiting time at nodes esc $\mathrm{C}_{3}$ and esc $_{5}$ (beginning of escalator of Line 8) and going downward from node $\mathrm{esc}_{3}$ to node $\mathrm{esc}_{5}$ and $\mathrm{esc}_{5}$ to $\mathrm{esc}_{6}$, respectively.

\section{Results}

Because passenger flow at platform entering into Line 3 was not overcrowded, constrained measurements have to be put in use in Line 8 . Firstly, the travel time for various passenger densities will be calculated with (3) and the route with minimum utility function will be picked up. According to (1), (8), and (9), the utility function of route during time interval 7:45-8:45 can be calculated as Table 5 shows.

As a result, the minimum utility function route is $e_{4} \rightarrow$ $\mathrm{esc}_{5} \rightarrow$ esc $_{6} \rightarrow P_{8}$; the route is chosen, and restricted measures on the node $e_{4}$ and esc $_{5}$ will be in action at the time interval of 7:45-8:45. According to (8), utility function of escalator $\left(\mathrm{esc}_{5}-\mathrm{esc}_{6}\right)$ is the minimum; therefore, decision points will be set prior to the escalator. Escalator $\left(\mathrm{esc}_{5}-\right.$ $\mathrm{esc}_{6}$ ) will be closed to increase utility function of route from entrance to platform.

If the escalator is closed, passengers are prone to choose another escalator rather than stairs to enter platform. Waiting time prior to escalator will be increased up to twice, but passenger volumes decline a little. Moreover, all escalators are closed, passengers have to choose the stairs, and the waiting time is increased further. The passenger flow at the platform will be diminished further. During time interval 8:00-9:15 AM, the passenger flow remains below 800 .

\section{Conclusions}

Firstly, this paper explained the characteristics of passengers and the general rules of passenger flow during peak periods from the perspective of walking time. And then the pedestrian walking path selection mechanism and the principles of path choice in transfer stations were analyzed. The abstract structure of station pedestrians has been established according to the control requirements during peak periods. The paper put forward the model assumptions under the consideration of passenger flow characteristics during peak periods. Based on that, the paper established passenger path control model during peak periods, and then the objective function of the passenger path choice control was determined. The effect factors of passenger route choice were studied in transfer station during rush hours. After that, based on the structure and layout of transfer station, the network of facilities was established according to the bottleneck identification and passenger volume during peak period. Finally, the paper established route planning model for passenger during peak periods. From the results, passenger crowdedness and passenger distribution at facilities models will be established to estimate the vulnerability and optimize layout of subway stations.

\section{Conflict of Interests}

The authors declare that there is no conflict of interests regarding the publication of this paper. 


\section{Acknowledgment}

This study is supported by the Fundamental Research Funds for the Central Universities (2014JBM152).

\section{References}

[1] W. Daamen and S. P. Hoogendoorn, "Level difference impacts in passenger route choice modelling," in Proceedings of the TRAIL Conference Proceedings, pp. 103-127, Delft, The Netherlands, 2004.

[2] W. Daamen, P. H. L. Bovy, and S. P. Hoogendoorn, "Influence of changes in level on passenger route choice in railway stations," Transportation Research Record, vol. 1930, no. 1, pp. 12-20, 2005.

[3] W. Daamen, Modelling Passenger Flows in Public Transport Facilities, TRAIL Thesis Series, Delft University Press, 2004.

[4] D. Helbing, L. Buzna, A. Johansson, and T. Werner, "Self-organized pedestrian crowd dynamics: experiments, simulations, and design solutions," Transportation Science, vol. 39, no. 1, pp. 1-24, 2005.

[5] T. Kretz, A. Grünebohm, A. Kessel, H. Klüpfel, T. Meyer-König, and M. Schreckenberg, "Upstairs walking speed distributions on a long stairway," Safety Science, vol. 46, no. 1, pp. 72-78, 2008.

[6] R. Nagai, M. Fukamachi, and T. Nagatani, "Evacuation of crawlers and walkers from corridor through an exit," Physica A: Statistical Mechanics and its Applications, vol. 367, pp. 449-460, 2006.

[7] D. R. Parisi and C. O. Dorso, "Microscopic dynamics of pedestrian evacuation," Physica A: Statistical Mechanics and its Applications, vol. 354, no. 1-4, pp. 606-618, 2005.

[8] L. Wang, Q. Zhang, Y. Cai, J. Zhang, and Q. Ma, "Simulation study of pedestrian flow in a station hall during the Spring Festival travel rush," Physica A: Statistical Mechanics and Its Applications, vol. 392, no. 10, pp. 2470-2478, 2013.

[9] R. Zhang and H. Zhao, "Study on pedestrian speed characteristics for passage of urban passenger transit hubs," in Proceedings of the International Conference on Remote Sensing, Environment and Transportation Engineering (RSETE '11), pp. 1679-1682, IEEE, June 2011.

[10] T. Nagatani, "Dynamical transition and scaling in a mean-field model of pedestrian flow at a bottleneck," Physica A: Statistical Mechanics and Its Applications, vol. 300, no. 3-4, pp. 558-566, 2001.

[11] J. Zhang, W. Klingsch, T. Rupprecht, A. Schadschneider, and A. Seyfried, "Empirical study of turning and merging of pedestrian streams in T-junction," http://arxiv.org/abs/1112.5299.

[12] R. Y. Guo and H. J. Huang, "A mobile lattice gas model for simulating pedestrian evacuation," Physica A: Statistical Mechanics and its Applications, vol. 387, no. 2-3, pp. 580-586, 2008.

[13] X. Guo, J. Chen, S. You, and J. Wei, "Modeling of pedestrian evacuation under fire emergency based on an extended heterogeneous lattice gas model," Physica A: Statistical Mechanics and its Applications, vol. 392, no. 9, pp. 1994-2006, 2013.

[14] X. Guo, J. Chen, Y. Zheng, and J. Wei, "A heterogeneous lattice gas model for simulating pedestrian evacuation," Physica A: Statistical Mechanics and its Applications, vol. 391, no. 3, pp. 582592, 2012.

[15] H. Cheng and X. Yang, "Emergency evacuation capacity of subway stations," Procedia-Social and Behavioral Sciences, vol. 43, pp. 339-348, 2012.
[16] X. Sun, Y. Zhang, G. Qin, H. Dong, and F. Guan, "Pedestrian transfer time optimization of urban rail transit based on ACP approach," in Proceedings of the IEEE International Conference on Automation and Logistics (ICAL '12), pp. 90-95, Zhengzhou, China, August 2012.

[17] F. Kaakai, S. Hayat, and A. El Moudni, "A hybrid Petri netsbased simulation model for evaluating the design of railway transit stations," Simulation Modelling Practice and Theory, vol. 15, no. 8, pp. 935-969, 2007.

[18] S. P. Hoogendoorn and W. Daamen, "Design assessment of Lisbon transfer stations using microscopic pedestrian simulation," in Proceedings of the 9th Computers in Railways Congress (CompRail '04), pp. 135-147, 2004.

[19] J. Y. S. Lee, H. I. William, and S. C. Wong, "Pedestrian simulation model for Hong Kong underground stations," in Proceedings of the IEEE Conference on Intelligent Transportation Systems, pp. 554-558, 2001.

[20] C. Y. Cheung and W. H. K. Lam, "Pedestrian route choices between escalator and stairway in MTR stations," Journal of Transportation Engineering, vol. 124, no. 3, pp. 277-285, 1998.

[21] W. H. K. Lam and C.-Y. Cheung, "Pedestrian speed/flow relationships for walking facilities in Hong Kong," Journal of Transportation Engineering, vol. 126, no. 4, pp. 343-349, 2000.

[22] S. P. Hoogendoorn and P. H. L. Bovy, "Pedestrian route-choice and activity scheduling theory and models," Transportation Research B: Methodological, vol. 38, no. 2, pp. 169-190, 2004.

[23] D. Hoffman, S. Murthy, and A. Khamisy, "System and method for a quality of service in a multi-layer network element," Google Patents, 2000.

[24] X. Rao, "An analysis of passenger delays in stairs and escalators of uban rail transit station," Traffic \& Transportation, no. 1, pp. 13-15, 2005 (Chinese).

[25] S. C. Wong, W. L. Leung, S. H. Chan et al., "Bidirectional pedestrian stream model with oblique intersecting angle," Journal of Transportation Engineering, vol. 136, no. 3, pp. 234-242, 2010.

[26] W. Xie, Passenger control scheme for urban rail transit transfer station [Master dissertation], Jiaotong University, Beijing, China, 2012, (Chinese).

[27] Y. Bi and S. Jiang, "On the indicator system of rail transit station platform space service," Urban Rail Transit Research, vol. 10, pp. 7-10, 2013.

[28] R. Tsuchiya, Y. Sugiyama, K. Yamauchi, K. Fujinami, R. Arisawa, and T. Nakagawa, "Route-choice support system for passengers in the face of unexpected disturbance of train operations," in Proceedings of the 10th International Conference on Computer System Design and Operation in the Railway and Other Transit Systems (COMPRAIL '06), pp. 189-197, July 2006. 


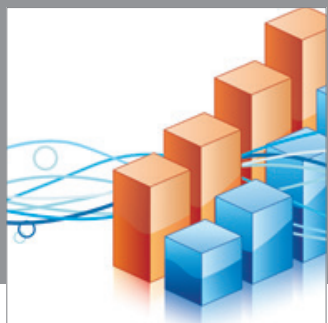

Advances in

Operations Research

mansans

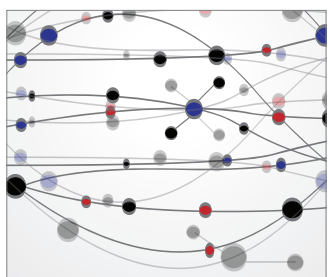

The Scientific World Journal
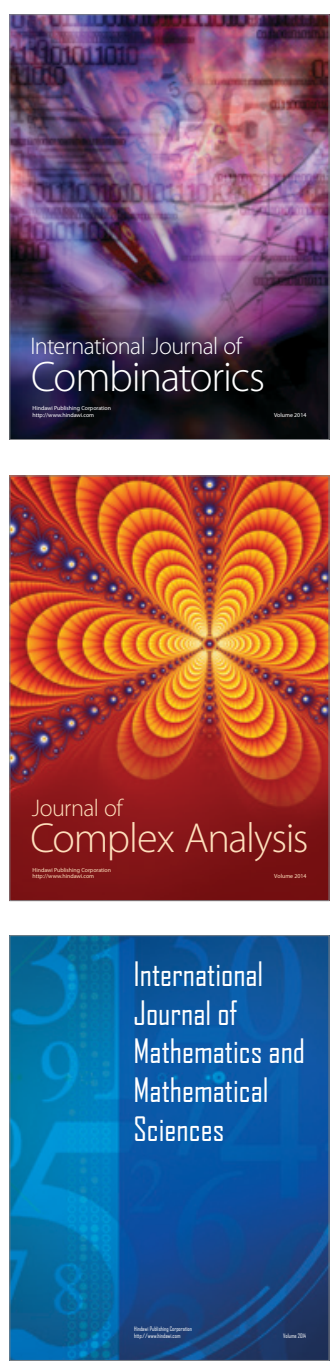
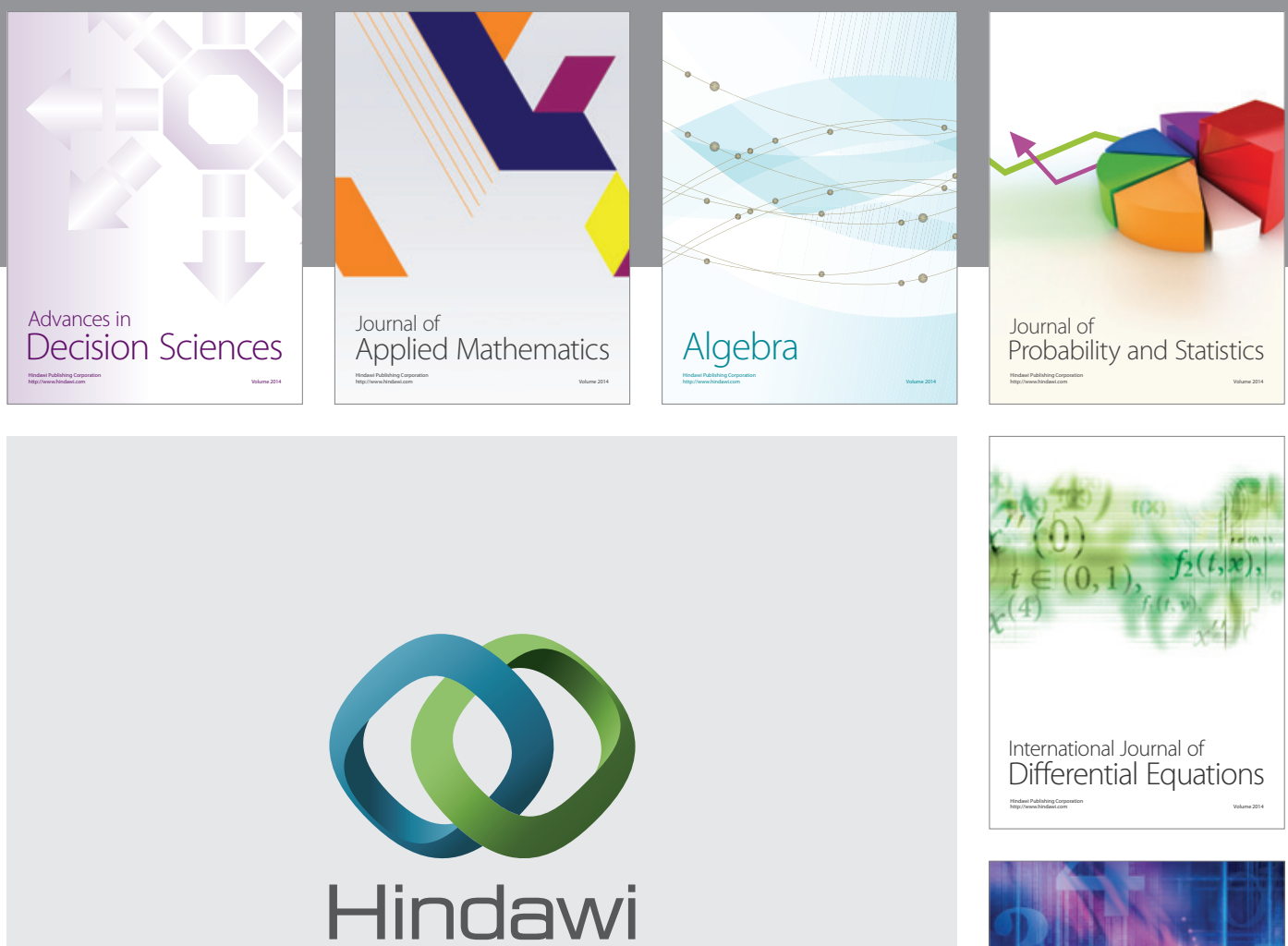

Submit your manuscripts at http://www.hindawi.com
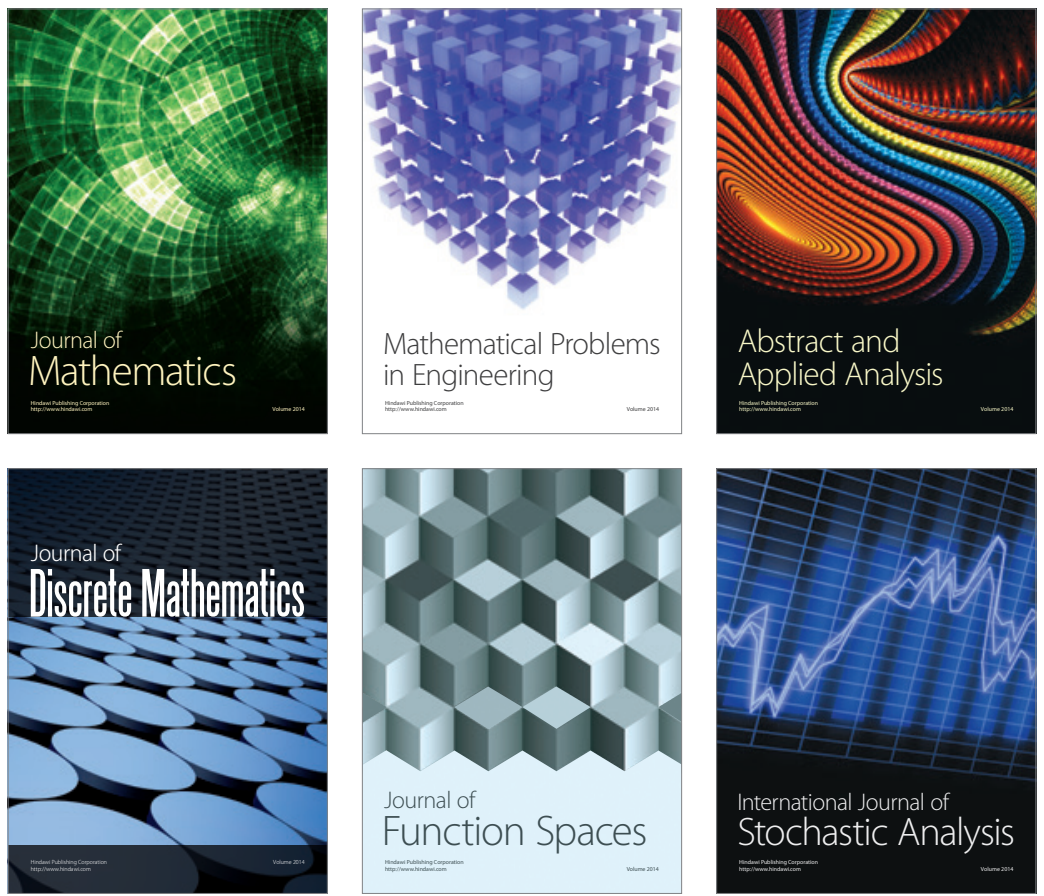

Journal of

Function Spaces

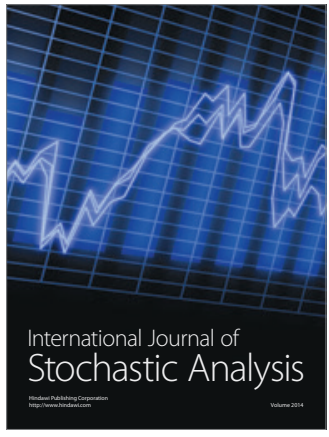

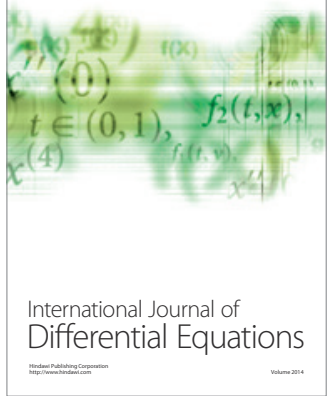
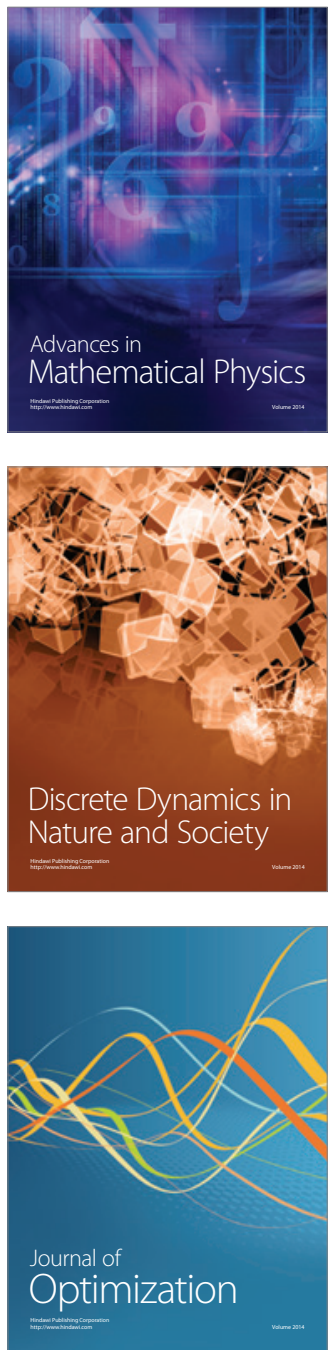